$(13 / 52)$ of UU-infected men $(P=0.04)$. Among doxycycline-treated men, failure occurred in $24.1 \%(7 / 29)$ and $30.9 \%(17 / 55)$, respectively $(\mathrm{P}=0.64)$. At 6 weeks, $80 \%(4 / 5)$ of UP and $58 \%(7 / 12)$ of UU-infected men originally treated with doxycycline and re-treated with azithromycin were failures; failure among men originally treated with azithromycin and re-treated with doxycycline was $57 \%(8 / 14)$ and $55 \%(6 / 11)$, respectively. At 9 weeks, failure after treatment with moxifloxacin occurred in $27.3 \%(3 / 11)$ of UP and $36.4 \%(4 / 11)$ of UU-infected men.

Conclusion Azithromycin was less effective against UP than UU. Failure after re-treatment with alternate therapy and moxifloxacin was common for both UU and UP.

\section{P2.104 ACCEPTABILITY OF CARRAGUARD VAGINAL GEL USE AMONG UGANDAN COUPLES (VIRGINAL MICROBICIDE ACCEPTABILITY)}

doi:10.1136/sextrans-2013-051184.0368

P Kimbowa. Center for HIV/STD and TB prevention, Kampala, Uganda

Objectives To evaluate the acceptability of candidate microbicide Carraguard among couples participating in a safety trial.

Study Design A 6-month randomised, placebo - controlled trial was conducted in active, low-risk couples in Uganda.

Methods Couples who were monogamous, HIV uninfected, and not regular condom users were enrolled. Acceptability data were collected through structured question at repeated intervals. At the closing study visit, participants were asked questions about hypothetical product characteristics and future use. Compliance with gel use was assessed by questionnaires, coital diaries, and tracking of used and unused applicators.

Results Among 55 enrolled couples, follow up and adherence with gel use were high and sustained, with $80 \%$ of women using gel in over $95 \%$ of vaginal sex acts. Because acceptability results from Carraguard and placebo arms were similar, they were combined for this analysis. Overall, $92 \%$ of women and $83 \%$ of men liked the gel somewhat or very much; $66 \%$ of women and $72 \%$ of men reported increased sexual pleasure with gel use; and $55 \%$ of women and $62 \%$ of men reported increased frequency of intercourse. Only $15 \%$ of women but $43 \%$ of men thought that gel could be used without the man knowing. Although men and women had similar views overall, concordance within couples was low, with no kappa coefficients above 0.31 .

Conclusion Carraguard gel use was acceptable to low-risk couples in western Uganda. Reported associations between gel use and increased sexual pleasure and frequency suggest a potential to market microbicide products for both disease prevention and enhancement of pleasure.

\section{P2.105 PUBLIC HEALTH PROVIDERS' PERCEPTIONS OF PARTNER NOTIFICATION FOR CHLAMYDIA TRACHOMATIS: BARRIERS AND FACILITATORS}

doi:10.1136/sextrans-2013-051184.0369

${ }^{1}$ K A T M Theunissen, ${ }^{1 P}$ Schipper, ${ }^{1,2} \mathrm{C}$ J P A Hoebe, ${ }^{3} \mathrm{R}$ Crutzen, ${ }^{1,2} \mathrm{~N}$ H T M DukersMuijrers. 'Dept. of Sexual Health, Infectious Diseases and Environmental Health. Public Health Service South Limburg, Geleen, The Netherlands; 'Department of Medical Microbiology Maastricht Infection Centre (MINC), School for Public Health and Primary Care (CAPHRI), Maastricht University Medical Centre (MUMC+), Maastricht, The Netherlands; ${ }^{3}$ Department of Health Promotion, School for Public Health and Primary Care (CAPHRI), Maastricht University Medical Centre $(M U M C+$ ), Maastricht, The Netherlands

Background In practise partner notification (PN) is suboptimal, because of several barriers such as time pressure, lack of financial reimbursement and provider discomfort. Perceptions of PN have mostly been studied in the field of medicine (i.e., among general practitioners (GPs) and medical specialists). This study assesses the barriers and facilitators of the application of PN among public health care providers (i.e., STI clinics), which is where the majority of PN occurs.

Methods Between March and June 2012, semi-structured interviews were conducted with 22 public health care providers from 6 of the 8 national STI clinics in The Netherlands.

Results All public health care providers reported feeling comfortable discussing $\mathrm{PN}$ and preferred patient referral (even though it is less effective than provider referral). Facilitators of PN included time, one-on-one consultations and motivational interviewing techniques. Important barriers to PN were the lack of feedback regarding its effectiveness and regarding the motivational strategies that were used. Furthermore, an emphasis on individuals and their autonomy leads to reduced feelings of responsibility towards the at risk-community (the scope of public health care).

Conclusion Because of existing barriers in the PN process, public health STI clinics do not reach their potential to protect the vulnerable community. Our results provide insight into the challenges at the patient, provider and organisational levels and can be used to optimise the $\mathrm{PN}$ process.

\section{P2.106 UTILIZATION OF SEXUALLY TRANSMITTED INFECTION SERVICES BY MALE PARTNERS OF ANTENATAL CARE ATTENDEES IN JINJA DISTRICT-UGANDA: A CASE CONTROL STUDY}

doi:10.1136/sextrans-2013-051184.0370

R Kisa, A Mukose, F Makumbi. Makerere University, Kampala, Uganda

Background Although men are key players in reproductive health services, their health seeking behaviours are poor. Sexually transmitted infections (STIs) are associated with increased spontaneous abortions, Human Immunodeficiency virus infection and infertility in pregnant women but men hardly utilise STI services when their partners seek antenatal care (ANC). In 2009/2010, men constituted 20\% of patients who received treatment for STIs in Jinja district. Only $3.9 \%$ of ANC attendees in Jinja hospital went with their male partners and utilised STI services between February and August 2011.

Objective To determine factors associated with utilisation of STI services by male partners of ANC attendees in Jinja district, Uganda. Methods A Case control study was conducted in 2012 on a consecutive sample of; 151 cases (Male partners of ANC attendees who utilised STI services at the health facility with their pregnant partners) and 151 controls (Male partners of ANC attendees who did not utilise STI services at the health facility with their pregnant partners) from 13 health facilities. Quantitative data was collected using semi-structured standardised questionnaires, entered in EPI INFO and analysed in stata/SE 10.0 using odds ratios from logistic regression models to assess associations. Qualitative data was collected by in-depth interviews and analysed using manifest content analysis.

Results Cases had higher odds of; prior STI testing (AOR 4.03, CI $2.22,7.32$ ), receipt of STI information (AOR 4.94, CI 2.50, 9.75) and being satisfied with the level of confidentiality at the health facilities (AOR 4.51, CI 2.16, 9.42). Fear of STI tests, lack of trust for their female pregnant partners, inadequate staff and busy schedules bar men from utilising STI services.

Conclusion Knowledge about STIs through prior STIs testing or Information Education and Communication materials as well as patients' satisfaction with health providers' handling of confidential information are important determinants of STI service utilisation.

\section{P2.107 ATOPIC DERMATITIS IN A HIV-POSITIVE COHORT AT RUHR UNIVERSITY BOCHUM}

doi:10.1136/sextrans-2013-051184.0371

${ }^{1} \mathrm{C}$ Tigges, ${ }^{2} \mathbf{W}$ Fuchs, ${ }^{2} \mathrm{~A}$ V Potthoff, ${ }^{2} \mathrm{~N}$ H Brockmeyer. 'Helios Klinik, Oberhausen, Germany; ${ }^{2}$ St. Josef Hospital, Bochum, Germany 